\title{
Defective in vivo expression and apparently normal in vitro expression of a newly identified 105-kDa lower lamina lucida protein in dystrophic epidermolysis bullosa
}

\author{
L.S.CHAN, ${ }^{*} \dagger$ J.-D.FINE, $\ddagger$ C.HAMMERBERG, ${ }^{*}$ E.A.BAUER $§$ AND K.D.COOPER* \\ *Departments of Dermatology, University of Michigan School of Medicine, Ann Arbor, MI. U.S.A. \\ †Northwestern University Medical School, Chicago, IL, U.S.A. \\ $\ddagger$ University of North Carolina School of Medicine, Chapel Hill, NC, U.S.A. \\ §Stanford University School of Medicine, Stanford, CA, U.S.A. \\ Accepted for publication 2 August 1994
}

Summary We have previously identified a novel 105-kDa lower lamina lucida protein detected by the autoantibodies from a group of patients who developed a unique immune-mediated subepidermal bullous dermatosis. We sought to determine if this novel basement membrane zone (BMZ) protein is normally expressed in the skin of patients with various subsets of epidermolysis bullosa (EB). Indirect immunofluorescence microscopy performed on non-lesional skin sections from patients with three major $\mathrm{EB}$ subsets revealed absence or significantly reduced expression of this novel BMZ protein in 20 out of 23 skin sections from patients with generalized dominant and recessive dystrophic EB. However, immunoblot analyses with the autoantibodies on Western-blotted proteins revealed that a comigrating 105-kDa protein is present in both cytosol extracts $(n=6)$ and conditioned media $(n=$ 3 ) of cultured dermal fibroblasts derived from patients with dystrophic EB, as well as those cultured from two healthy individuals. Although the reason for such disparate findings is not known, the defective in vivo expression of this novel 105-kDa protein in dystrophic EB is presumably not due to a failure of fibroblasts to synthesize or secrete the protein. It is possible, however, that the 105-kDa protein may be unable to incorporate into the BMZ because it is produced in a dysfunctional form, or its BMZ binding site is missing. It is also possible that other structural alterations in skin BMZ, which occur in dystrophic $\mathrm{EB}$, result in masking of the antigenic binding by the autoantibody when intact BMZ is probed. In any case, the reduced in vivo expression of the $105-\mathrm{kDa}$ protein represents additional evidence for a defect in BMZ composition in dystrophic $\mathrm{EB}$ which extends to a number of molecular components.

Hereditary epidermolysis bullosa (EB) consists of a heterogeneous group of skin diseases manifested by the development of blisters at sites of minor mechanical trauma. ${ }^{1}$ EB subtypes are classified by clinical phenotypic features, mode of inheritance, level of skin cleavage, and the presence or absence of additional ultrastructural or immunohistochemical findings. ${ }^{1}$ Because sufficient phenotypic overlap exists among all three major subtypes of EB clinically, precise classification usually requires the performance of either transmission electron microscopy or immunofluorescence mapping. ${ }^{1,2}$

Correspondence: L.S.Chan, Northwestern Univ. Dept. of Dermatology, Tarry 4-711, Mail Code T225, 300 E. Superior Street, Chicago, IL 60611-3010, U.S.A.

Presented in part at the Annual Meeting of the Society for Investigative Dermatology, Baltimore, MD, U.S.A., 29 April-2 May 1992.
EB simplex is histologically characterized by intraepidermal blister formation, ${ }^{1}$ and point mutations of keratin 14 or keratin 5 in some of its subtypes. ${ }^{3.4}$ No basement membrane zone (BMZ) component defect has been consistently demonstrated in $\mathrm{EB}$ simplex skin. ${ }^{1}$ Junctional EB is histologically characterized by intra-lamina lucida blister formation, ${ }^{1}$ associated in some patients with morphometric abnormalities in hemidesmosomes and sub-basal dense plates. Although defects in two lamina lucida components, nicein (GB3, BM-600, kalinin, epiligrin) and 19-DEJ-1 antigen, have been described, ${ }^{5,7}$ the definitive genetic defect(s) has not yet been determined. Dystrophic EB is histologically characterized by sublamina densa blister formation and, in some forms, with either reduction or absence of anchoring 
fibrils. ${ }^{1}$ It is now believed that all forms of dystrophic EB are caused by abnormalities in expression of type VII collagen, although altered tissue staining has in general been noted in cases of generalized recessive dystrophic EB. ${ }^{8-11}$ Genetic linkage studies also support a genetic defect of type VII collagen in dominant dystrophic EB. ${ }^{12,13}$ Although a defect in type VII collagen, a major component of anchoring fibrils $^{14}$ at the sublamina densa site of pathological separation, is likely to be the primary pathogenic mechanism in dystrophic $\mathrm{EB}$, four other $\mathrm{BMZ}$ components (19-DEJ-1, KF-1, AF1/AF2, and chondroitin-6sulphate proteoglycan) have also been reported to be defective in varying degrees in dystrophic $\mathrm{EB}$ skin. ${ }^{5,15-17}$ The full extent of dysfunctional BMZ formation in dystrophic EB, however, remains to be determined.

We have recently identified and partially characterized a novel 105-kDa lower lamina lucida autoantigen, by using autoantibodies from a unique group of patients who developed an erythema multiforme/toxic epidermal necrolysis-like immune-mediated bullous dermatosis. ${ }^{18-20}$ This 105-kDa BMZ component has been localized by immunoelectron microscopy to the lower portion of the lamina lucida. The autoantibodies recognized only a $105-\mathrm{kDa}$ protein band present in cytosol and culture media of both normal human keratinocytes and fibroblasts; hence, this protein is synthesized and secreted by both normal human keratinocytes and fibroblasts. ${ }^{18}$ By comparative immunoblot analyses, comparative immunomapping studies, and two-dimensional gel electrophoreses, this 105-kDa BMZ protein has been shown to be an acidic protein, distinct from the laminin family and the kalinin B2t chain. ${ }^{21}$ Although this component is not present in fetal skin up to 20 weeks' gestation, it is present in neonatal skin. ${ }^{18}$

We report the in vivo expression of this novel BMZ component in the skin of patients with dystrophic EB, and its in vitro expression in cultured fibroblasts derived from patients with dystrophic EB.

\section{Methods}

\section{Patient selection}

Study subjects were patients with EB who were enrollees in the National EB Registry. Diagnosis and subclassification were based on recently published criteria. ${ }^{1}$ Normal controls included specimens of healthy adult skin and neonatal foreskin.

\section{Indirect immunofluorescence study}

Cryosections $(6 \mu \mathrm{m})$ of skin biopsies from EB patients and normal control subjects were prepared. Cryosections were first incubated with the serum $(1: 10$ dilution) from a unique patient with autoantibodies directed against a lower lamina lucida determinant, ${ }^{18}$ followed by incubation with fluorescein-conjugated goat antihuman IgG. ${ }^{18}$ The sections were examined with a Nikon Optiphot microscope by epi-illumination.

\section{Fibroblast cultures}

Dermal fibroblast cultures from two healthy adults and one patient with dominant dystrophic EB were derived and maintained as previously described. ${ }^{22}$ The fibroblasts were grown and subpassaged in Dulbecco's modified Eagle's medium (DMEM) (GIBCO-BRL, Grand Island, NY, U.S.A.) supplemented with $10 \%$ fetal bovine serum (Hyclone, Logan, UT, U.S.A.), 1\% penicillin/ streptomycin, and $5 \mathrm{mmol} / \mathrm{l} \mathrm{L}$-glutamine, at $37^{\circ} \mathrm{C}$. Confluent monolayers of cells were subpassaged weekly. Two fibroblast cell lines (GM 10245, GM 10388), derived from patients with a clinical diagnosis of recessive dystrophic $\mathrm{EB}$, were obtained from the National Institute of General Medical Sciences Human Genetic Mutant Cell Repository (Camden, NJ, U.S.A.). Three other fibroblast cell lines $(80585,81645,79461)$ were derived from patients with severe recessive dystrophic EB. Other fibroblast cell lines were derived from a patient with EB simplex (81681) and a patient with junctional EB. The clinical diagnoses of all the EB patients from whom the fibroblast cell lines were derived were confirmed by electron microscopic examination.

\section{Immunoblot analyses}

At confluency, cultured cells were washed three times with Hanks' balanced salt solution, and then harvested with $2 \mathrm{mmol} / \mathrm{l}$ phenymethylsulphonyl fluoride in phosphate-buffered saline. The total cytosol proteins were obtained by subjecting the cell suspension to three cycles of freeze/thaw and centrifugation $\left(15,000 \mathrm{~g}, 4^{\circ} \mathrm{C}, 30 \mathrm{~min}\right)$. The cytosol proteins were further concentrated by centrifugation (5000 r.p.m., $4^{\circ} \mathrm{C}, 1 \mathrm{~h}$ ) with a $30-\mathrm{kDa}$ molecular weight cut-off concentrator (R.A.Grace, Beverly, MA, U.S.A.). Fibroblast conditioned media were obtained by the following method: at confluency, cells were washed three times with Hanks' balanced salt solution, and then incubated 
with serum-free DMEM for $6 \mathrm{~h}$. The conditioned media were then collected and filtered through a $0 \cdot 22 \mu \mathrm{m}$ filter (Millipore, Bedford, MA, U.S.A.) to eliminate cellular debris, and subsequently concentrated through a 30$\mathrm{kDa}$ cut-off concentrator, as described above. The total protein concentrations in the cytosol extracts and conditioned media were determined by a standard Bradford colorimetric protein assay (Bio-Rad, Hercules, CA, U.S.A.). An identical quantity of total fibroblast protein derived from each of the patients and normal controls was loaded on to the slots of a $4 \%$ stacking gel over a $7 \cdot 5 \%$ separating gel. ${ }^{18}$ Sodium dodecyl sulphatepolyacrylamide gel electrophoresis was performed under reducing conditions, as previously described. ${ }^{18}$ The separated proteins were then electrophoretically transferred to nitrocellulose paper (Bio-Rad). The nitrocellulose paper was blocked with $5 \%$ powdered milk in
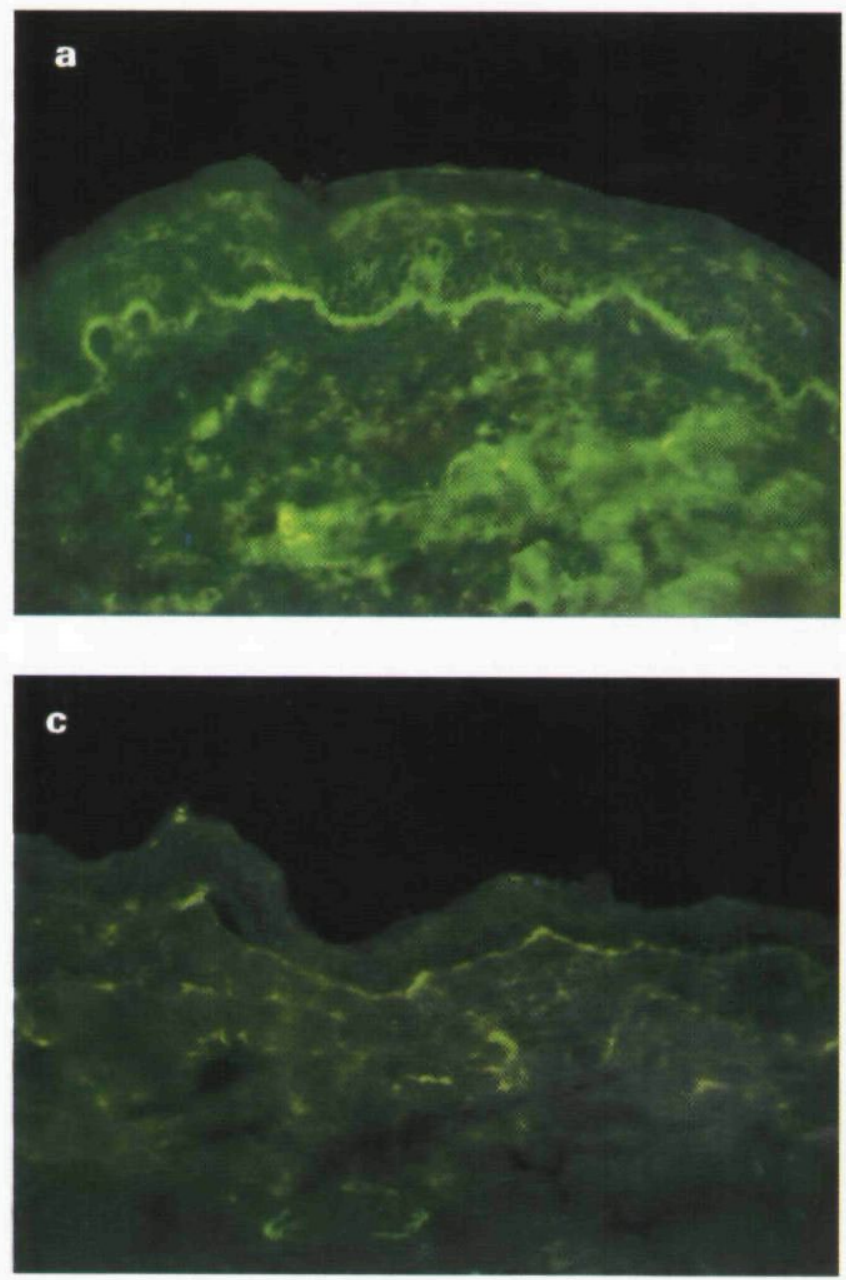

phosphate-buffered saline, and was then reacted with a $1: 100$ dilution of the serum from the patient who had autoantibodies directed against a 105-kDa fibroblast protein. ${ }^{18}$ The specific immunological reaction was visualized with peroxidase-conjugated goat antihuman IgG (Fc-specific, Caltag, South San Francisco, CA, U.S.A.) and a standardized substrate of $3,3^{\prime} 5,5^{\prime}$ tetramethylbenzidine (Kirkegaard \& Perry, Gaithersburg, MD, U.S.A.).

\section{Results}

In vivo expression of the $105-\mathrm{kDa}$ BMZ protein in skin sections

The results of indirect immunofluorescence studies performed with the autoantibodies on skin sections from
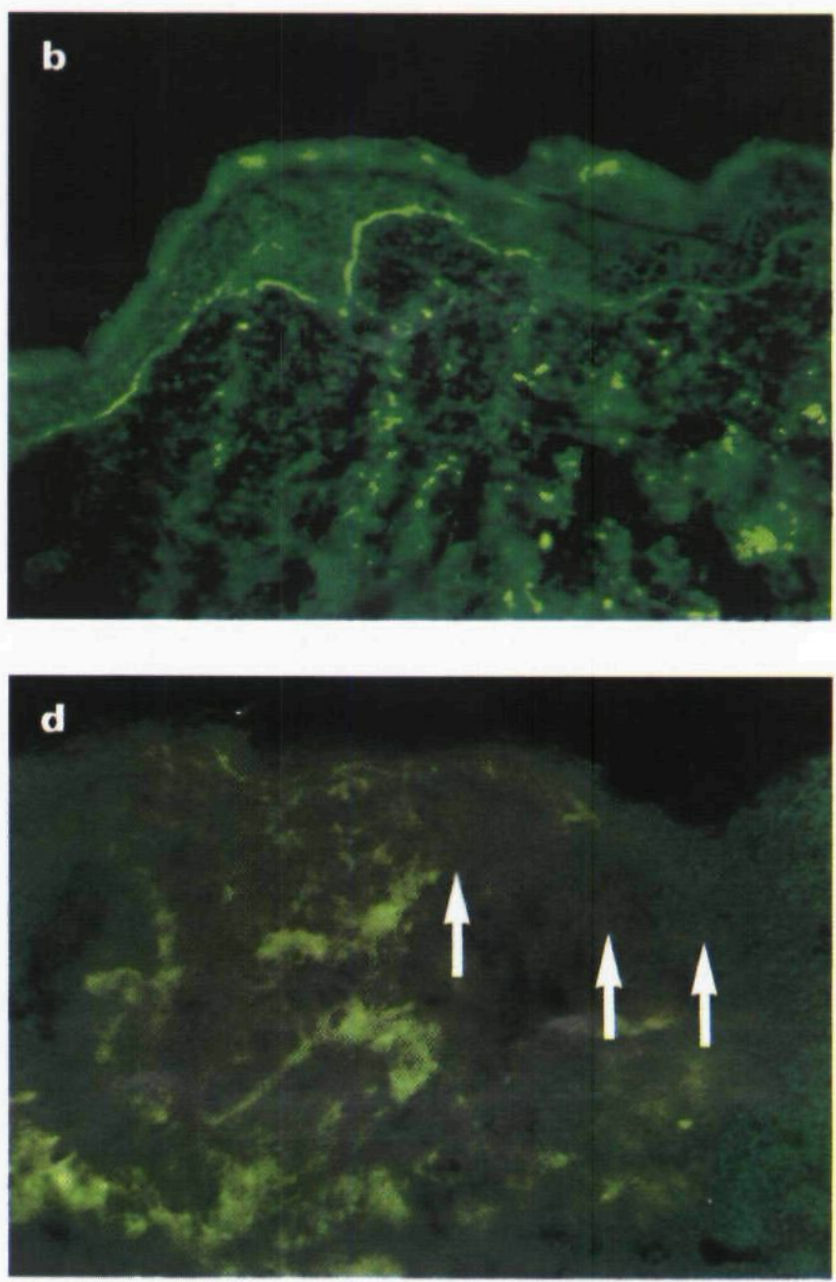

Figure 1. Examination of the $105 \mathrm{kDa}$ basement membrane zone (BMZ) protein expression in non-lesional skin obtained from normal individuals and from patients with various subsets of epidermolysis bullosa (EB), by reacting with the autoantibodies that detected a $105 \mathrm{kDa}$ BMZ protein, followed by fluorescein-conjugated goat anti-human IgG. (a) Normal skin; (b) EB simplex skin; (c) junctional EB skin; (d) dystrophic EB skin. Arrows indicate focal absence of BMZ protein. 
patients with EB subsets are described below. Representative samples of the study are shown in Figure 1. In all 10 normal control skin sections, the in vivo expression of the 105-kDa BMZ protein was a strong, linear, and continuous pattern along the BMZ (Fig. 1a). In all the skin sections from patients with $\mathrm{EB}$ simplex and junctional EB, the expression of the $105-\mathrm{kDa}$ BMZ protein was present (Fig. 1b and c). However, weaker and focally discontinuous expression was noted in three out of 10 and two out of 10 skin sections of patients with $\mathrm{EB}$ simplex and junctional EB, respectively. In contrast, there was a total absence of this BMZ protein expression in six out of 12 and seven out of 11 skin sections from patients with dominant and recessive dystrophic EB, respectively. In the remaining patients with dystrophic $\mathrm{EB}$, this $\mathrm{BMZ}$ protein expression was very weak in intensity, and there were many discontinuities (Fig. 1d). The skin sections from one patient with dominant dystrophic $\mathrm{EB}$ and one patient with recessive dystrophic $\mathrm{EB}$ had normal BMZ expression.

\section{In vitro expression of the 105-kDa protein in fibroblasts}

In order to examine whether or not the in vivo defective expression of this BMZ protein in skin sections of patients with dystrophic $\mathrm{EB}$ is due to a failure of fibroblasts to synthesize or secrete this protein, we next investigated the in vitro expression of this protein in dermal fibroblasts cultured from patients with dystrophic EB. When the Western-blotted proteins of equal quantity were reacted with the autoantibodies that identified a $105-\mathrm{kDa}$ protein, a $105-\mathrm{kDa}$ protein was detected in both cytosol extracts and conditioned media of fibroblasts cultured from three dystrophic EB patients (one dominant form, two recessive form), as well as those cultured from two healthy individuals

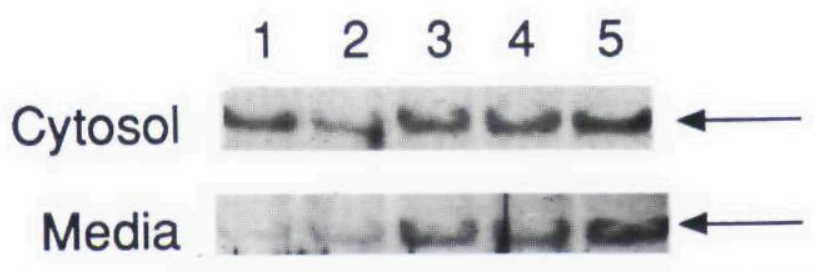

Figure 2. Western immunoblotting of cytosol extracts (upper panel) and serum-free conditioned media (lower panel) of cultured fibroblasts derived from two healthy individuals (lanes 1 and 2), a patient with dominant dystrophic epidermolysis bullosa (EB) (lane 3), and two patients with recessive dystrophic EB (lanes 4 and 5, GM 10245 and GM 10388). The Western blots of equal quantities of total proteins were reacted with the autoantibodies which identified a $105 \mathrm{kDa}$ protein (arrows), and visualized with peroxidase-conjugated goat anti-human IgG.
(Fig. 2). The positive in vitro expression of this $105-\mathrm{kDa}$ protein was further confirmed by immunoblotting against fibroblast cytosol extracts derived from three other patients with recessive dystrophic EB, one patient with $\mathrm{EB}$ simplex, and one patient with junctional $\mathrm{EB}$ (data not shown).

\section{Discussion}

Our results suggest that in vivo defective expression of this $105-\mathrm{kDa}$ BMZ protein in non-lesional skin sections of patients with dystrophic EB is not due to a failure of fibroblasts to synthesize or secrete the protein, as it is readily detectable in an in vitro assay of cell culture supernatant and cytosol extracts. One possible, but unlikely, explanation is that the protein may be produced in a dysfunctional form, rendering it incapable of incorporating into the BMZ. However, the amino acid composition of the dystrophic EB fibroblast protein, particularly the epitope(s) recognized by our antisera, must not have been altered significantly, as it remained as immunoreactive as that derived from normal fibroblasts (Fig. 2). In addition, such a mutation of the dystrophic EB fibroblast protein would have to be subtle, because it retains the same molecular size as the normal fibroblast protein (Fig. 2).

Another possibility, which is not examined by the present study, is that the defective in vivo expression of this $105-\mathrm{kDa}$ lamina lucida component is the result of keratinocyte dysfunction and/or damage, assuming that keratinocytes play a more important role in the formation of this component of the basement membrane. However, this possibility seems unlikely, as fibroblasts and keratinocytes synthesize and secrete this protein in the same amount, ${ }^{20}$ suggesting that they contribute equally to its formation.

A third possibility is that the abnormal binding which we have observed reflects masking of the epitopes recognized by our antisera, as a result of a disordered spatial configuration of the BMZ, due to the absence of or altered structure of another critical skin BMZ component, most likely type VII collagen. Other candidates include KF-1, chondroitin-6-sulphate proteoglycan, and AF1/AF2.

A fourth possibility is that the binding site for this novel $105-\mathrm{kDa}$ protein may be missing in the skin of patients with generalized dystrophic EB. One such candidate is type VII collagen, which is known to be abnormally expressed or mutated in dystrophic EB skin.

Reduced, but positive, in vivo expression of this $105 \mathrm{kDa}$ BMZ protein can also be observed in the skin 
of a minority of patients with two other subtypes of $\mathrm{EB}, \mathrm{EB}$ simplex and junctional EB. The reason for such decreased in vivo expression is not clear, but is not without precedent. In particular, 19-DEJ-1 antigen, a hemidesmosome-anchoring filament complex-associated antigen, is undetected in about $25 \%$ of recessive dystrophic $\mathrm{EB}$ specimens, ${ }^{5}$ and is absent in all junctional EB skin. ${ }^{6}$ The in vivo expression of another lamina lucida antigen, nicein (GB3), is reduced in the skin of patients with the indeterminate form of junctional $\mathrm{EB}$, and is virtually absent in the skin of patients with the lethal form of junctional EB. ${ }^{7,23}$ The relationships between this $105 \mathrm{kDa}$ protein and 19-DEJ-1 and nicein remain to be determined. Further studies, including biochemical purification of this novel 105-kDa BMZ protein, and molecular cloning of the cDNA encoding for the protein, may further elucidate these complex structural and functional relationships within the skin BMZ.

\section{Acknowledgments}

This work was supported in part by Dermatology Foundation (Annerberg Foundation Career Development Award, L.S.Chan), NIH NIAMS contracts N01 AR 62271, AR22201, AR22204, and AR22200, grants RO1 AR34861 (J.-D.Fine), R37AR19537 and P01AR41045 (E.A.Bauer), and NIH Clinical Investigator Award (K.D.Cooper). The authors thank Dr YueYing Liu (Northwestern University) for her technical assistance.

\section{References}

1 Fine J-D, Bauer EA, Briggaman RA et al. Revised clinical and laboratory criteria for subtypes of inherited epidermolysis bullosa: a consensus report by the subcommittee on diagnosis and classification of the National Epidermolysis Bullosa Registry. J Am Acad Dermatol 1991; 24: 119-35.

2 Hintner H, Stingl G, Schuler G et al. Immunofluorescence mapping of antigenic determinants within the dermal-epidermal junction in mechanobullous diseases. J Invest Dermatol 1981; 76: 113-18.

3 Coulombe PA, Hutton ME, Letai A et al. Point mutations in human keratin 14 genes of epidermolysis bullosa simplex patients: genetic and functional analyses. Cell 1991; 66: 1301-11.

4 Bonifas JM, Rothman A-L, Epstein EH. Epidermolysis bullosa simplex: evidence in two families for keratin gene abnormalities. Science 1991; 254: 1202-5.

5 Fine J-D, Horiguchi Y, Couchman JR. 19-DEJ-1, a hemidesmosomeanchoring filament complex-associated monoclonal antibody: definition of a new skin basement membrane antigenic defect in junctional and dystrophic epidermolysis bullosa. Arch Dermatol 1989; 125: 520-3.

6 Fine J-D. 19-DEJ-1, a monoclonal antibody to the hemidesmosomeanchoring filament complex, is the only reliable immunohistochemical probe for all major forms of junctional epidermolysis bullosa. Arch Dermatol 1990; 126: 1187-90.
7 Heagerty AHM, Kennedy AR, Eady RAJ et al. GB3 monoclonal antibody for diagnosis of junctional epidermolysis bullosa. Lancet 1986 ; i: 860 .

8 Heagerty AHM, Kennedy AR, Leigh IM et al. Identification of an epidermal basement membrane defect in recessive forms of dystrophic epidermolysis bullosa by LH7:2 monoclonal antibody: use in diagnosis. Br J Dermatol 1986; 115: 125-31.

9 Leigh IM, Eady RAJ, Heagerty AHM et al. Type VII collagen is a normal component of epidermal basement membrane, which shows altered expression in recessive dystrophic epidermolysis bullosa. J Invest Dermatol 1988; 90: 639-42.

10 Rusenko KW, Gammon WR, Fine J-D, Briggaman RA. The carboxyl-terminal domain of type VII collagen is present at the basement membrane in recessive dystrophic epidermolysis bullosa. J Invest Dermatol 1989; 92: 623-7.

11 Parente MG, Chung LC, Ryynänen J et al. Human type VII collagen: cDNA cloning and chromosomal mapping of the gene. Proc Natl Acad Sci USA 1991; 88: 6931-5.

12 Ryynänen M, Ryynänen J, Sollberg S et al. Genetic linkage of type VII collagen (COL7A1) to dominant dystrophic epidermolysis bullosa in families with abnormal anchoring fibrils. J Clin Invest 1992; 89: 974-80.

13 Ryynänen M, Knowlton RG, Parente MG et al. Human type VII collagen: genetic linkage of the gene (COL7A1) on chromosome 3 to dominant dystrophic epidermolysis bullosa. Am J Hum Genet 1991; 49: 797-803.

14 Sakai LY, Keene DR, Morris NP, Burgeson RE. Type VII collagen is a major structural component of anchoring fibrils. J Cell Biol 1986; 103: 1577-86.

15 Fine J-D, Breathnach SM, Hintner H, Katz SI. KF-1 monoclonal antibody defines a specific basement membrane antigen defect in dystrophic forms of epidermolysis bullosa. J Invest Dermatol 1984; 82: 35-8.

16 Goldsmith LA, Briggaman RA. Monoclonal antibodies to anchoring fibrils for the diagnosis of epidermolysis bullosa. J Invest Dermatol 1983; 81: 464-6.

17 Fine J-D, Couchman JR. Chondroitin 6-sulfate proteoglycan but not heparan sulfate proteoglycan is abnormally expressed in skin basement membrane from patients with dominant and recessive dystrophic epidermolysis bullosa. J Invest Dermatol 1989; 92: 611-16.

18 Chan LS, Fine J-D, Briggaman RA et al. Identification and partial characterization of a novel 105-kilodalton lower lamina lucida autoantigen associated with a novel immune-mediated subepidermal blistering disease. J Invest Dermatol 1993; 101: 262-7.

19 Chan LS, Cooper KD. A novel immune-mediated subepidermal bullous dermatosis characterized by IgG autoantibodies to a lower lamina lucida component. Arch Dermatol 1994; 130: 343-7.

20 Cotell SL, Lapiere JC, Chen JD et al. A novel 105-kDa basement membrane autoantigen: association with bullous pemphigoid. J Invest Dermatol 1994; 103: 78-83.

21 Chan LS, Lapiere J-C, Marinkovich MP et al. The newly identified $105-\mathrm{kD}$ lower lamina lucida autoantigen is an acidic protein distinct from the 105-kD $\gamma 2$ chains of laminin-5. J Invest Dermatol (in press).

22 Chan LS, Hammerberg C, Kang K et al. Human dermal fibroblast interleukin-1 receptor antagonist (IL-1ra) and interleukin-1 $\beta$ $(\amalg-1 \beta)$ mRNA and protein are co-stimulated by phorbol ester: implication for a homeostatic mechanism. J Invest Dermatol 1992; 99: 315-22.

23 Domloge-Hultsch N, Gammon WR, Briggaman RA et al. Epiligrin, the major human keratinocyte integrin ligand, is a target in both an acquired autoimmune and an inherited subepidermal blistering skin disease. J Clin Invest 1992; 90: 1628-33. 
This document is a scanned copy of a printed document. No warranty is given about the accuracy of the copy. Users should refer to the original published version of the material. 